\title{
Automatic Morse Code Recognition Under Low SNR
}

\author{
Xianyu Wang ${ }^{\mathrm{a}}$, Qi Zhao ${ }^{\mathrm{b}}$, Cheng $\mathrm{Ma}^{\mathrm{c},{ }^{*}}$ and Jianping Xiong ${ }^{\mathrm{d}}$ \\ Department of Precision Instrument, Tsinghua University, Beijing 100084, China; \\ allwswxy@163.com, bzhao-q16@mails.tsinghua.edu.cn, cmacheng@mail.tsinghua.edu.cn, \\ dxiongjp@tsinghua.edu.cn
}

Keywords: Morse code, automatic recognition, deep learning

\begin{abstract}
As an important means of communication, Morse has a wide range of applications in life such as medical treatment, radio broadcasts and so on. However, there has been no systematic method for automatic recognition of Morse codes. This paper designs a Morse code automatic recognition system that combines signal processing and deep learning algorithms. Through experimental analysis of each part of the system, an algorithm suitable for Morse was found and a better result was achieved.
\end{abstract}

\section{Introduction}

Morse, the main use of short-wave wireless communications, is one of the important means of communication [1]. The advantages of Morse include simple coding methods, strong noisy immunity, ease of implementation. With the rapid development of communication technology, Morse Communications has been greatly affected, slowly withdraw from the stage of history. However, they are still active in some areas of civilian communications such as radio broadcasts, aviation announcements, maritime communications, combat readiness maneuvers and so on [2].

The Morse telegram is directly encoded and sent out through the transmitter [3], but the majority of the receiving and decoding work is still carried out by manual. With the interference of various industries and personal wireless communications as well as the changes in the atmospheric, the electromagnetic environment is getting worse and worse nowadays which brings great difficulty and challenge to the decoding. Along with the increasing number of information, a large number of high-quality operator is required. What's more, the heavy and repetitive work in a strong noisy environment can easily lead to the fatigue of the operator, resulting in the situation of mistranslation and the missing translation. Therefore, it is necessary and valuable to develop an automatic Morse code recognition system [4].

Since the 1970s, the automatic Morse code recognition technology had become a hot topic that many research institutions at home and abroad have done research on this [5-8]. The recognition is not difficult to achieve for high signal-to-noise Morse telegraph signals. However, when the signal-to-noise ratio is very low and the background noise has a high energy, this brings great difficulties to signal recognition in the traditional sense. It is a new research direction to apply machine learning method to Morse code recognition. At present, some papers using SVM, k-means, clustering algorithm for Morse code recognition [9-11] and get better results. However, when the types of the actual signal code are not enough or the signal-to-noise ratio is strong, the accuracy of the algorithm decreases. In this paper, a new approach developed to solve the problem of Morse code recognition is presented that combined with signal processing and speech recognition.

The rest of this paper is organized as follows. The background of Morse code recognition is introduced in Section 2. Section 3 focuses on the experiment procedures and evaluation. Section 4 includes the discussion of this paper. The paper ends with our conclusion and suggests future work. 


\section{Background of Morse Code Recognition}

\subsection{Morse Code}

The Morse code is a time series, invented by Morse in 1844. It expresses the corresponding English letters, numbers and punctuation marks in different order. The code includes five kinds: dot, dash, dot space, character space, and word space. The tone ratio of dot to dash has to be 1:3 and the silent ratio (dot-space: character-space: word space) has to be 1:3:5 based on the definition of Morse code. The standard Morse spectrum is shown in Figure 1.

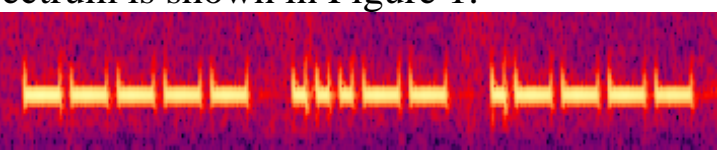

Figure 1. Standard Morse spectrum

However, due to the influence of various electromagnetic environments, the Morse signal is usually of poor quality.

\subsection{Feature Extraction}

Before recognition, the Morse signal first needs feature extraction to provide a mathematical basis for subsequent recognition algorithms.

\subsubsection{Mel-Frequency Cepstral Coefficients}

Do Mel frequency is based on the auditory characteristics of the human ear, and it has a nonlinear relationship with $\mathrm{Hz}$ frequency. Mel Frequency Cepstral Coefficient, calculated using the relationship between Mel Frequency and $\mathrm{Hz}$ frequency, is mainly used for voice data feature extraction and reducing the computational dimension. The process of extracting MFCC is shown in Figure 2. [12].

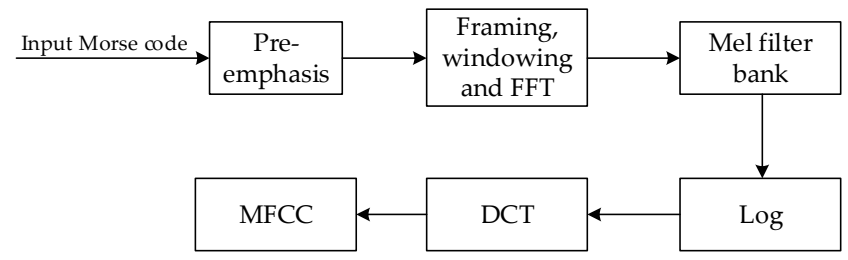

Figure 2. Process of extracting MFCC

\subsubsection{Improved Feature Extraction Algorithm}

Unlike speech signal, the Morse signal is more concentrated, and the requirements on the frequency bandwidth characteristics are not very high. Aiming at the characteristics of Morse signal, an improved feature extraction algorithm is proposed. The process of improved feature extraction algorithm is shown in Figure 3.

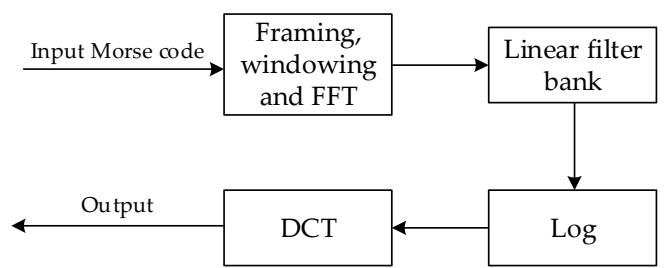

Figure 3. Process of improved feature extraction algorithm

Compared to the MFCC, the pre-emphasis process is removed and the Mel filter bank is changed to a linear filter bank. The center frequency is $1 \mathrm{KHz}$, the bandpass filter frequency range is set to $800 \sim 1200 \mathrm{KHz}$, and the bandpass filter group number is 13 . The linear filter bank structure is shown in Figure 4.

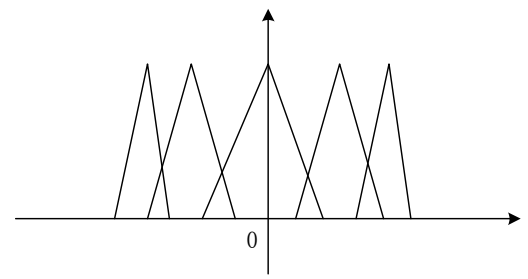

Figure 4. Linear filter bank structure 
The filter bandwidth near the center frequency is the widest and narrower toward both sides.

\subsection{Recognition Algorithm}

\subsubsection{Hidden Markov Model}

Hidden Markov model is a statistical Markov model that has powerful temporal pattern classification ability. Therefore, it is suitable for time series modeling of dynamic processes and in theory it can handle timing patterns of any length. HMM has a wide range of applications such as speech recognition, handwriting recognition, image texture modeling and classification. [13]

HMM is a double stochastic process on the basis of the Markov chain. One of these is the Morkov chain, which is a basic stochastic process that describes the transfer of states. Another stochastic process describes the statistical correspondence between states and observations. From the observer's point of view, only the observation value can be seen. The presence and characteristics of the state are perceived through a random process, and therefore it is called a hidden Markov model.

\subsubsection{Gaussian Mixture Model}

The Gaussian mixture model is an extension of the single Gaussian probability density function and can approximate the density distribution of any shape smoothly. Similar to clustering, according to Gaussian probability density function (PDF) parameters, each Gaussian model can be regarded as a category. Entering a sample x, it can be calculated by PDF, and then judged by a threshold whether the sample belongs to a Gaussian model. GMM is suitable for multi-classification and can be applied to complex object model. [14]

\subsubsection{Deep Belief Network}

The deep belief network is a probabilistic generation model. Compared with the traditional neural network of the discriminant model, the generative model establishes a joint distribution between observation data and labels. [15]

DBNs consist of multiple Restricted Boltzmann Machines layers. The networks are limited to one visible layer and one hidden layer. There are connections between layers, but no connections between the elements in the layers. Hidden layer units are trained to capture the correlation of high-order data that appears in the visible layer.

\section{Experiment Procedures and Evaluation}

\subsection{System Structure}

The structure of the system is shown in the figure 5.

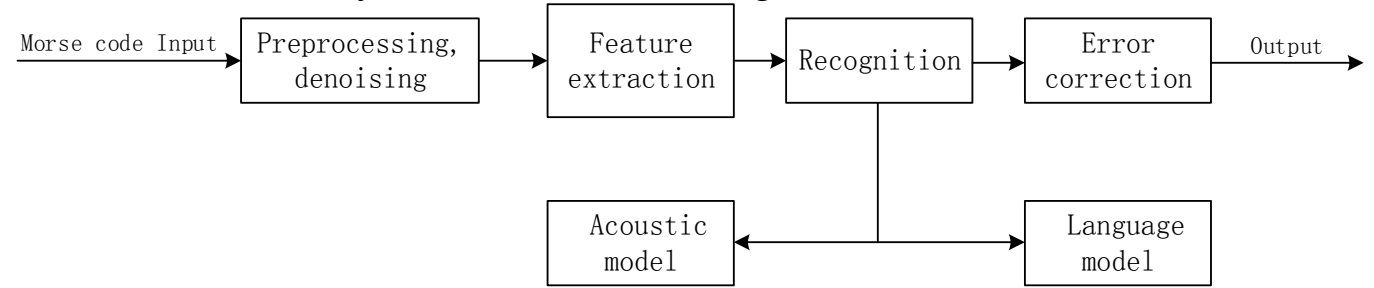

Figure 5. Classic DBN network structure

\subsection{Database}

In Morse code recognition, there is no universal database. The database used in this article is produced by software and the total number of data is 2020. The composition is shown in table 1 .

Table 1. The composition of database.

\begin{tabular}{ccc}
\hline & SNR & amount \\
\hline Train & $8 \mathrm{db}$ & 1860 \\
Test & $8 \mathrm{db}$ & 80 \\
Testn & $-3 \mathrm{db}$ & 80 \\
\hline
\end{tabular}

There are two ways to label the Morse code, labeling by dot and dash or by word. When using the single-factor-based GMM-HMM method for recognition, the recognition results of the two methods are shown in table 2 . 
Table 2. Recognition results

\begin{tabular}{ccc}
\hline & Wer(8db) & Wer(-3db) \\
\hline By dot and dash & $110 \%$ & $143 \%$ \\
By word & $35 \%$ & $56 \%$ \\
\hline
\end{tabular}

In the first case, the basic unit of recognition and the output is dot and dash. Usually, the length of dot and dash is not completely fixed. At the same time, if truncated, it is easy to cause misidentification. As you can see, the results of the experiment exceeded $100 \%$ due to a lot of insertion errors.

In the second case, the output is combined into words according to the results of dot and dash recognition. The wrong combination can be effectively ruled out, thus solving the problem that the duration is not fixed. Follow-up experiment is based on the second case.

\subsection{HMM State Number}

The number of states of the HMM represents the correspondence between specific sound signals and labels. When using the single-factor-based GMM-HMM method for recognition, the recognition results of different HMM state number are shown in table 3.

Table 3. The recognition results of different HMM state number

\begin{tabular}{cc}
\hline HMM state number & Wer(8db) \\
\hline 4 & $40 \%$ \\
5 & $37 \%$ \\
6 & $35 \%$ \\
7 & $56 \%$ \\
\hline
\end{tabular}

As can be seen from the above table, when the number of states is 6 , the recognition result is the best. When the state is adjusted from four to six, the recognition rate does not increase significantly. However, when the number of states exceeds six, the recognition rate drops dramatically that a severe overfitting phenomenon occurs.

\subsection{Feature Extraction}

When using the single-factor-based GMM-HMM method for recognition, the recognition results of different feature extraction algorithm are shown in table 4.

Table 4. The recognition results of different feature extraction algorithm

\begin{tabular}{ccc}
\hline feature extraction algorithm & Wer(8db) & Wer(-3db) \\
\hline MFCC & $35 \%$ & $56 \%$ \\
Improved algorithm & $12 \%$ & $22 \%$ \\
\hline
\end{tabular}

MFCC is mainly based on the human ear's perception of frequency, and therefore covers a wide range of frequencies. In speech recognition, the corresponding features can be accurately extracted. However, for the Morse signal, the frequency band is usually narrow, and the requirement for feature extraction is relatively low in terms of frequency.

\subsection{Recognition Algorithm}

When using different recognition algorithms, the recognition results are shown in table 5.

Table 5. The recognition results of different recognition algorithms

\begin{tabular}{ccc}
\hline feature extraction algorithm & Wer(8db) & Wer(-3db) \\
\hline Monophone GMM-HMM & $12 \%$ & $22 \%$ \\
Triphone GMM-HMM & $3.4 \%$ & $26 \%$ \\
DNN-HMM & $1.1 \%$ & $18 \%$ \\
\hline
\end{tabular}

It can be seen that DNN has the highest method recognition rate in several methods. 


\section{Conclusions and Discussion}

Re In the work of this paper, we used the deep learning algorithms to implement the construction of the Morse identification system and achieved better results.

At present, the method based on deep learning is mainly driven by data. The quality of the database will directly affect the accuracy of the experiment. In the experiment process, many factors such as the balance and representativeness of data need to be considered. In the previous experiment, because the settings of the database did not reach equilibrium, many seemingly simple corpus was not properly identified.

In the course of DNN experiments, we found that the current mainstream deep learning algorithms has better robustness. In the actual environment, due to the influence of truncation and annihilation factors, many signals sound very confusing, but they can be identified better by DNN method.

However, there is room for improvement in the current system. The recognition algorithms can be updated to introduce attention mechanisms, which will help the detection of endpoints. Besides, error correction algorithms also need to be improved.

\section{References}

[1]. Pawar P Y, Bhansali D S, Borate S R, et al. Smart phone Application using Morse Code and Inaudible Frequency. International Journal on Computer Science \& Engineering, 2013, 5(3).

[2]. King T W. Modern Morse code in rehabilitation and education: new applications in assistive technology. Allyn and Bacon, 2000.

[3]. Windle C. Variations in sending Morse code. Journal of Experimental Psychology, 1955, 50(1):75.

[4]. D. Finley, Morse Code: Breaking the Barrier, MFJ Publishing Company, Starkville, MS, 1997

[5]. Shih C H, Luo C H. A Morse-code recognition system with LMS and matching algorithms for persons with disabilities. International Journal of Medical Informatics, 1997, 44(3):193-202.

[6]. Hsieh M C, Luo C H, Mao C W. Unstable Morse code recognition with adaptive variable-ratio threshold prediction for physically disabled persons. IEEE Transactions on Rehabilitation Engineering A Publication of the IEEE Engineering in Medicine \& Biology Society, 2000, 8(3):405.

[7]. Yang C H, Luo C H, Jeang Y L, et al. A novel approach to adaptive Morse code recognition for disabled persons. Mathematics \& Computers in Simulation, 2000, 54(1-3):23-32.

[8]. Gold B. Machine recognition of hand-sent Morse code. Information Theory Ire Transactions on, 1959, 5(1):17-24.

[9]. Wu C M, Luo C H. Morse code recognition system with fuzzy algorithm for disabled persons. Journal of Medical Engineering \& Technology, 2002, 26(5):202.

[10]. Yang C H, Jin L C, Chuang L Y. Fuzzy support vector machines for adaptive Morse code recognition. Medical Engineering \& Physics, 2006, 28(9):925.

[11]. DUU-TONG FUH, CHING-HSING LUO. UNSTABLE MORSE CODE RECOGNITION SYSTEM WITH EXPERT-GATING NEURAL NETWORK. Biomedical Engineering Applications Basis \& Communications, 2002, 14(01):12-19.

[12]. Han W, Chan C F, Choy C S, et al. An efficient MFCC extraction method in speech recognition. IEEE International Symposium on Circuits and Systems, 2006. ISCAS 2006. Proceedings. IEEE, 2006:4 pp. 
[13]. Tokuda K, Yoshimura T, Masuko T, et al. Speech parameter generation algorithms for HMM-based speech synthesis. IEEE International Conference on Acoustics, Speech, and Signal Processing, 2000. ICASSP '00. Proceedings. IEEE, 2002:1315-1318.

[14]. Richard Blundell, Stephen Bond. GMM Estimation with persistent panel data: an application to production functions. Econometric Reviews, 2000, 19(3):321-340.

[15]. Hinton G, Deng L, Yu D, et al. Deep Neural Networks for Acoustic Modeling in Speech Recognition: The Shared Views of Four Research Groups. IEEE Signal Processing Magazine, 2012, 29(6):82-97. 